\title{
Cardiovascular Injury Due to SARS-CoV-2
}

\author{
Christina L. Bugert ${ }^{1} \cdot$ Victoria Kwiat $^{2} \cdot$ Isela C. Valera ${ }^{2} \cdot$ Joachim J. Bugert $^{3} \cdot$ Michelle S. Parvatiyar $^{2}$ (I)
}

Accepted: 29 January 2021 / Published online: 5 March 2021

(C) The Author(s), under exclusive licence to Springer Nature Switzerland AG part of Springer Nature 2021

\begin{abstract}
Purpose of Review The world is currently facing the largest global health crisis since the early 1900s due to a novel coronavirus. While SARS-CoV-2 infection causes predictable symptoms in COVID-19 patients, including upper respiratory distress and fever, the heterogeneity of manifestations is surprising. This review focuses on direct and indirect causes of myocardial injury in COVID-19 patients and highlights current knowledge, treatment strategies, and outstanding questions in the field.

Recent Findings Data are emerging that highlight the extent of cardiovascular involvement in COVID-19 patients, including evidence that SARS-CoV-2 causes myocarditis and increases cardiac risk. The incidence of cardiac injury is much greater in patients with severe disease presentation and those in intensive care.

Summary During the past year, COVID-19 patient mortality rates have improved due to tailored pharmacological treatments and patient management strategies that address the unique presentation of symptoms, which will hopefully also reduce the incidence of cardiac injury.
\end{abstract}

Keywords Cardiac injury $\cdot$ COVID-19 $\cdot$ Myocarditis $\cdot$ Arrhythmia $\cdot$ Cardiomyopathy

\section{Introduction}

Severe Acute Respiratory Syndrome coronavirus 2 (SARSCoV-2) represents a current public health crisis. The positive-sense single-stranded RNA virus, SARS-CoV-2, is a novel virus in the Coronaviridae subfamily and closely related to the genus Betacoronavirus [1]. Sars-CoV-2, which shares $79.6 \%$ sequence homology with Sars-CoV-1 [2] similarly infects hosts through the angiotensin-converting enzyme 2 (ACE2), the functional receptor in lung epithelial cells. For an illustration depicting viral entry see Fig. 1. The most common clinical manifestations of SARS-CoV-2 include fulminant pneumonia and acute respiratory distress syndrome

This article is part of the Topical Collection on Virology

Christina L. Bugert and Victoria Kwiat contributed as co-equal first authors

Michelle S. Parvatiyar

mparvatiyar@fsu.edu

1 College of Life and Environmental Sciences, University of Exeter, Exeter, South West, UK

2 Department of Nutrition, Food and Exercise Sciences, Florida State University, Tallahassee, FL, USA

3 Bundeswehr Institute of Microbiology, Munich, Germany
(ARDS) [3]. Infection of other cell types possessing ACE2 receptors has been described including the epithelial cells of the lung, intestine, kidney, heart, and blood vessels inducing cardiotropic damage $[4,5]$. The manifestation of tropism throughout the body has shown the unique complexity of this novel pathogen. Several new SARS-CoV-2 variants have recently been identified throughout the world including a new B.1.1.7 variant in the United Kingdom [6].

The disease caused by SARS-CoV-2 is now being understood as a multisystem infection and harms other organs besides the lungs. Therefore, Thus it is imperative that we understand the prolonged impact this illness may have on heart health in recovered patients. A growing number of reports have shown that COVID-19 patients are being hospitalized for lung-related symptoms yet dying of heart failure [7]. Therefore, it is of utmost importance to establish the frequency of infection of heart tissue in COVID-19 patients with multiple presentations of the disease. Since SARS-CoV-2 has marked affinity for the ACE2 receptor, it is certainly possible that the vascular endothelium and myocardium could be directly infected [8]. The heart can become damaged in COVID-19 by three distinct mechanisms: (i) the acute hyper-inflammatory response increases blood coagulability, which can cause blood clots that obstruct arteries and indirectly affect the heart. (Or the heart becomes damaged directly due to); (ii) by Infection of the heart muscle and the associated 


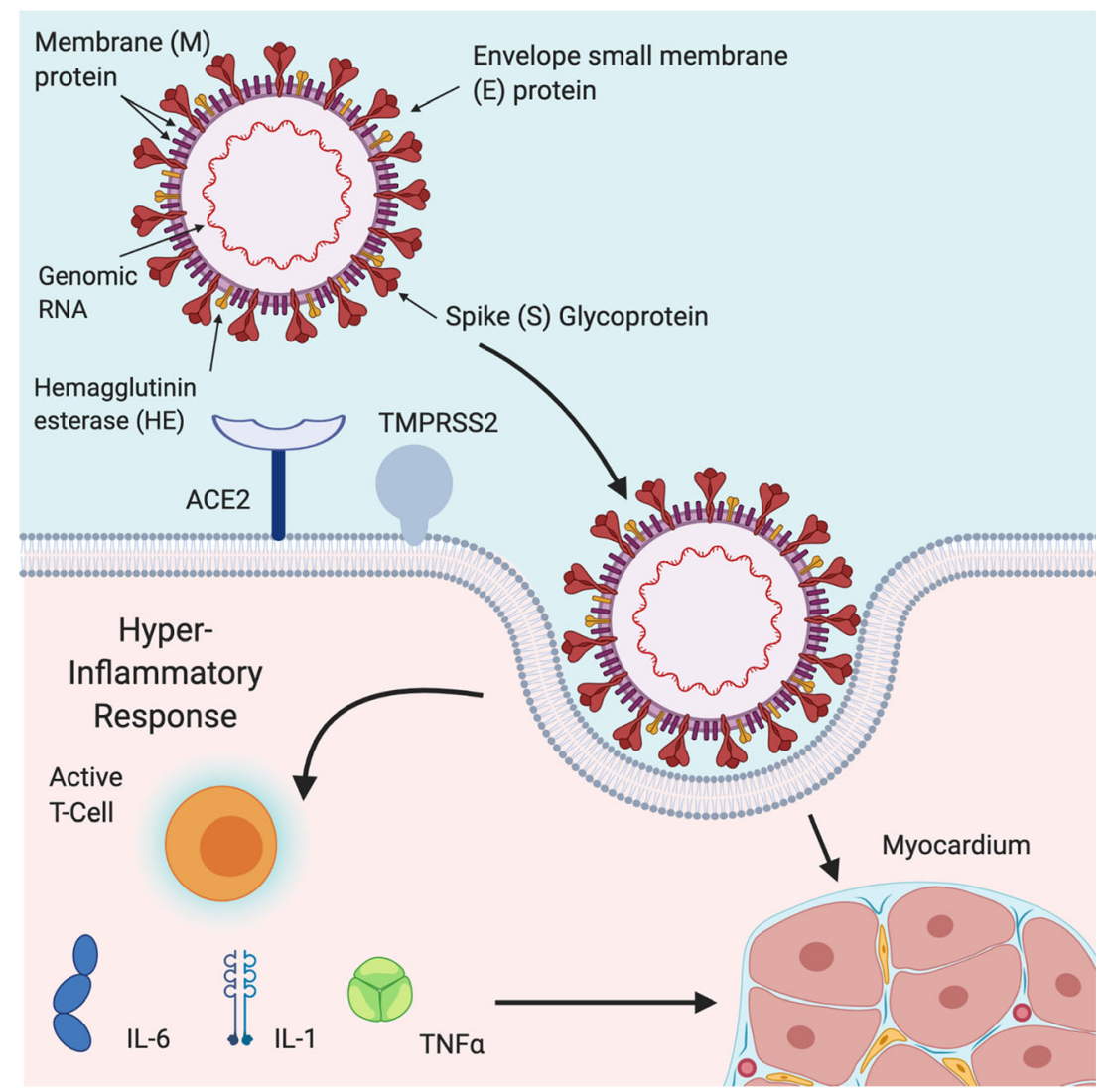

Fig. 1 Mechanism of SARS-CoV-2 Cellular. Entry into host cells is an important determinant of infectivity and infection and pathogenesis of viruses. SARS-CoV-2 has various proteins on its surface including (M) membrane protein, (E) envelope small membrane protein and (S) spike glycoproteins. Interaction of the SARS-CoV-2 spike protein with angiotensin-converting enzyme 2 (ACE2) receptors on target the cell surface mediates SARS-CoV-2 entry into cells that is facilitated by

inflammatory response; (iii) infection of vascular endothelium/ heart pericytes with subsequent local inflammation (vasculitis) and clot formation [9]. For further insight on mechanisms leading to cardiac injury, see Fig. 2.

Myocardial damage in COVID-19 patients [10] is evident when high levels of troponins are present in the blood and are significantly associated with death in hospitalized patients $[10,11]$. Early studies from Wuhan described heart failure as one of the most common complications seen in $24 \%$ of all patients and in $49 \%$ of patients who succumbed to the disease [3]. Heart failure can occur due to weakening of the heart muscle due to damage from systemic or localized inflammation or possibly due to infection. Therefore, SARSCoV-2 along with its myocardial implications appears to be a multifaceted health issue. In addition, the severity of the disease seemingly affects a subset of patients with comorbidities such as diabetes mellitus and hypertension [3]. Thus, the correlation between chronic damage to the cardiovascular system and infection by SARS-CoV-2 presents an additional challenge for clinicians. transmembrane protease serine 2 (TMPRSS2) and lysosomal proteases. Once SARS-CoV-2 enters the body it can elicit a hyper inflammatory response characterized by high levels of interleukin-6 (IL-6), interleukin1 (IL-1), tumor necrosis factor-alpha (TNF $\alpha)$ that can damage the myocardium or through direct infection of cardiomyocytes which can damage the heart. Activated T-cells are one of the immune cells involved in the antiviral response. Figure created using BloRender.com

\section{SARS-CoV-2 Infection and the Heart}

\section{Mechanism of SARS-CoV-2 Entry}

The mechanism of cardiac/cellular entry by SARS-CoV-2 begins with the binding of SARS-CoV-2 to the ACE2 receptor via the $\mathrm{S} 1$ region of the virus spike $(\mathrm{S})$ protein $[12,13]$. ACE2 is a membrane receptor that downregulates the proinflammatory cytokine, angiotensin II (Ang II) [12]. The S2 region of the S-protein facilitates fusion of the virus with host cell membranes followed by endocytosis of both SARS-CoV2 and ACE2 receptor into cells (see Fig. 1). This causes a general down regulation of ACE2 receptors leading to accumulation of Ang II in serum [12]. After infection, a cascade of stimulators induces various pro-inflammatory cytokines and chemokines such as TNF $\alpha$ and IL-6, which drive inflammation. While ACE2 is highly expressed in type 2 lung alveolar cells, single-cell RNA sequencing studies have shown that more than $7.5 \%$ of myocardial cells have positive ACE2 expression [14]. 


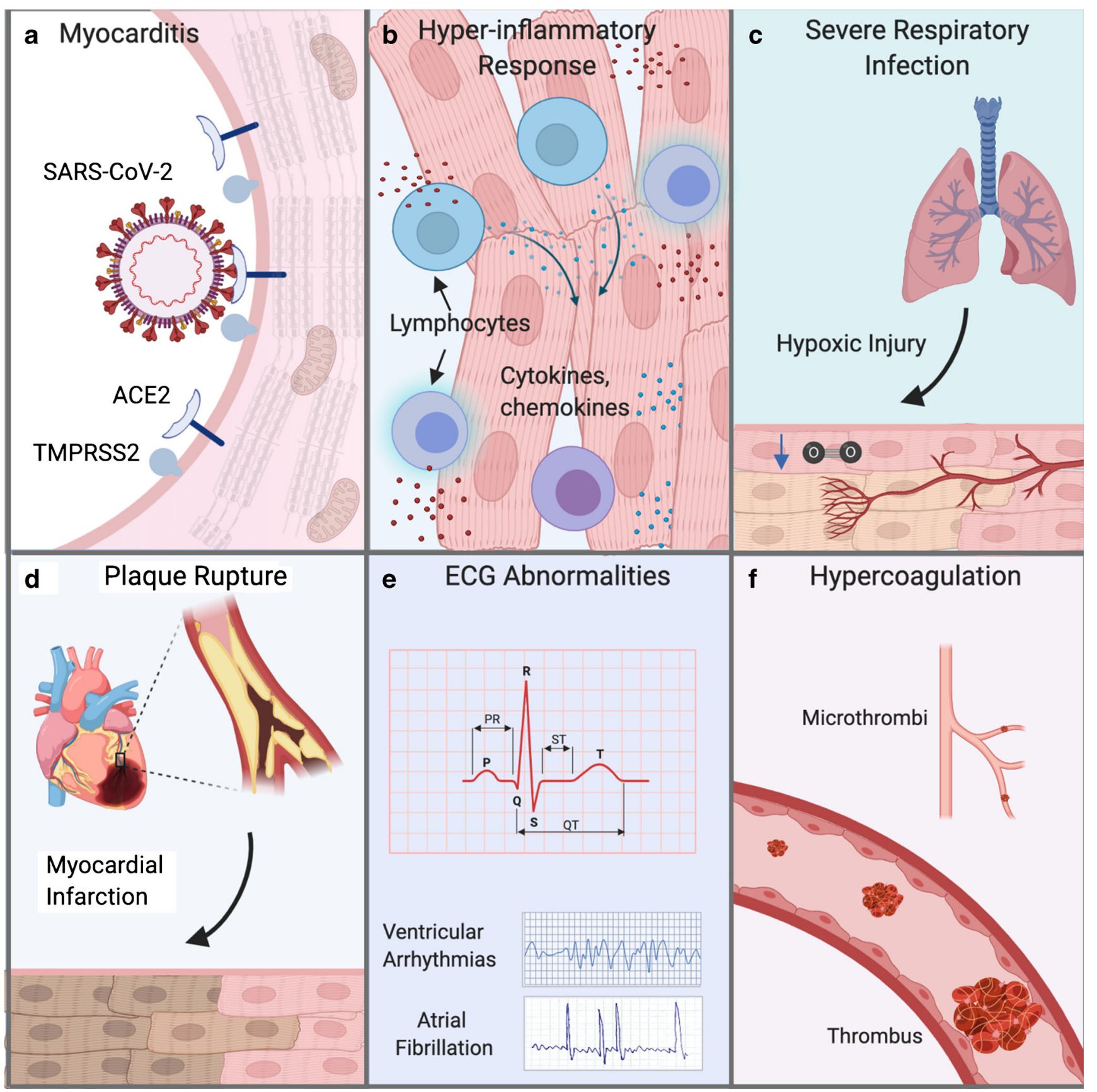

Fig. 2 Various Routes to Cardiac Injury Due to SARS-CoV-2 Infection. (A) Several studies suggest that SARS-CoV-2 can directly infect cardiac cells of the myocardium and may cause heart damage by a "direct effect". (B) The heart can also be injured by a systematic hyper inflammatory response that has been reported in COVID-19 patients by an "indirect effect". High levels of cytokines and chemokines can cause "bystander effects" and cardiac inflammation also classified as myocarditis or weakening of heart muscle function and may manifest as dilated cardiomyopathy (DCM) and/or heart failure. (C) Severe respiratory infection of SARS-CoV-2 infection can reduce pulmonary function and cause hypoxic injury in many organs including the heart due to low tissue oxygenation. (D) Increased stress on the cardiovascular system of
COVID-19 patients can worsen underlying cardiac risk factors including rupture of atherosclerotic plaques leading to ST-segment myocardial infarction (STEMI) myocardial infarction. COVID-19 patients with severe infection can also exhibit non-STEMI myocardial infarction. (E) Various alterations in heart rhythm may occur as the first cardiac symptom in COVID-19 patients and can manifest as ventricular arrhytmias and atrial fibrillation that are detectable by electrocardiogram (ECG). (F) Coagulation abnormalities lead to formation of thrombi that can block blood flow in larger vessels or microthrombi that occlude small blood vessels and capillaries to affect microcirculation. The location and size of the blood vessel blockage dictate extent of ischemic tissue damage. Figure created with BioRender.com 
It has been demonstrated that SARS-CoV-2 exhibits cardiac tropism similar to SARS-CoV and MERS-CoV. Some controversy exists over whether SARS-CoV-2 directly infects heart muscle cells, although several studies have identified viral particles by RT-PCR in adult endomyocardial biopsy specimens [15] and also cardiac macrophages [16]. An in vitro study confirmed the possibility of viral entry into cardiac cells using human induced pluripotent stem cellderived cardiomyocytes (hiPSC-CMs) utilizing microscopy coupled with RNA sequencing to demonstrate that SARSCoV-2 can gain entry into the cells via ACE2 [17].

\section{Cytokine Storm in Response to SARS-CoV-2 Infection}

Infection with coronaviruses SARS-CoV, MERS-CoV, and now SARS-CoV-2 has been shown to induce the immune system to release multiple cytokines and chemokines [18]. The innate immune response is the initial response to SARSCoV-2 infection (Fig. 3) and primes the adaptive immune response. This cytokine release syndrome can directly cause myocardial injury due to the hyper induction of pro-inflammatory cytokines including IL-1, IL-6, TNF $\alpha$, and IFN- $\gamma$. It has been previously documented that the heart is impacted by damaging inflammatory cascades that are generated in response to infection with coronaviruses and other viruses [19, 20]. The mechanisms of myocardial injury due to cytokine storm involve imbalance in type 1 and type 2 immune responses mediated by $\mathrm{T}$ helper cells [21, 22, 23]. Hyper-inflammatory shock has been reported in children with COVID-19 causing symptoms similar to Kawasaki disease that include coronary vessel abnormalities and cardiac dysfunction [24].

\section{Heart Damage Observed in COVID-19 Patients}

To date, clinical cardiovascular manifestations of COVID-19 include an elevation of cardiac biomarkers, cardiac arrhythmia, arterial and venous thromboembolism, and development of cardiogenic shock and cardiac arrest [25]. Initial reports that SARS-Co-V2 causes cardiac injury came out of Wuhan, China, and statistics from some studies reached as high as $23 \%$ of reported cases [21]. In patients admitted to the intensive care unit (ICU), biomarkers of cardiac injury including cardiac troponin I (cTnI) were significantly higher than in non-ICU patients. COVID-associated cardiac injuries demonstrate multiple etiologies that range from myocardial infarction (MI), myocarditis, sepsis-mediated myocardial injury, or stress-induced takotsubo cardiomyopathy.

Furthermore, it is challenging to differentiate the causes of cardiac injury in COVID-19 patients since underlying causes can be diverse, including acute coronary syndrome (ACS) caused by plaque rupture or thrombosis (type I MI) or supply-demand mismatch (type II MI), stress-induced cardiomyopathy, and cytokine release syndrome [25]. In addition, patients with cardiac injury exhibit increased signs of severe systemic inflammation including higher leukocyte counts, C-reactive protein, procalcitonin, and increased levels of myocardial injury biomarkers such as creatine kinase, NTproBNP, and myoglobin [26]. While other proposed mechanisms of damage may be attributed to ACE2-related signaling pathways including downregulation of ACE2 expression [27]. Dysfunction of the respiratory system causes hypoxemia or low blood oxygenation and is one mechanism that increases susceptibility of the myocardium to damage [27]. Initial hyper-inflammatory events and cytokine syndrome seen in severe cases of COVID-19 cause hypercoagulability and coronary microvascular thrombosis. This can cause coronary plaque rupture or supply-demand mismatch leading to myocardial ischemia/infarction that can also lead to cardiac damage [28].

\section{Co-Morbidities Impact Cardiac Risk}

According to a meta-analysis of 65,484 patients, the consensus was that individuals suffering from preexisting comorbidities had a greater risk of mortality during infection, particularly patients with cardiovascular disease indicating two-fold greater mortality risk [29]. Comorbidities such as cardiovascular disease and diabetes mellitus in SARS-CoV-1 patients presented an $8 \%$ and $11 \%$ rate of mortality, respectively [30]. This severity of infection and mortality was also implicated in individuals suffering from underlying diseases such as hypertension, diabetes mellitus, congestive heart failure, chronic kidney disease, and cancer [29]. Diabetic patients for example had a worse prognosis, which was attributed to poor metabolic control [31]. Furthermore, obesity which is also a comorbidity for many cardiovascular diseases leads to a compromised immune response and difficulties during mechanical ventilation [32].

\section{Cardiovascular Complications in COVID-19 Patients}

The presence of myocardial injury was associated with a significantly worse prognosis in patients in a study in China [3] indicated by increased blood troponin levels. In a study of 416 patients in ICU, 20\% had evidence of cardiac injury, which was associated with a five-fold increase in the need for mechanical ventilation [33]. In a study based upon a patient cohort in the Mount Sinai Health System, 36\% of the 2,736 patients had elevated troponin blood concentrations, and when adjusted for relevant clinical factors and severity of disease, even small amounts of myocardial injury (e.g., troponin I > $0.03-0.09 \mathrm{ng} / \mathrm{ml}$ ) were associated with a significantly higher risk of death [34]. A range of cardiovascular complications 


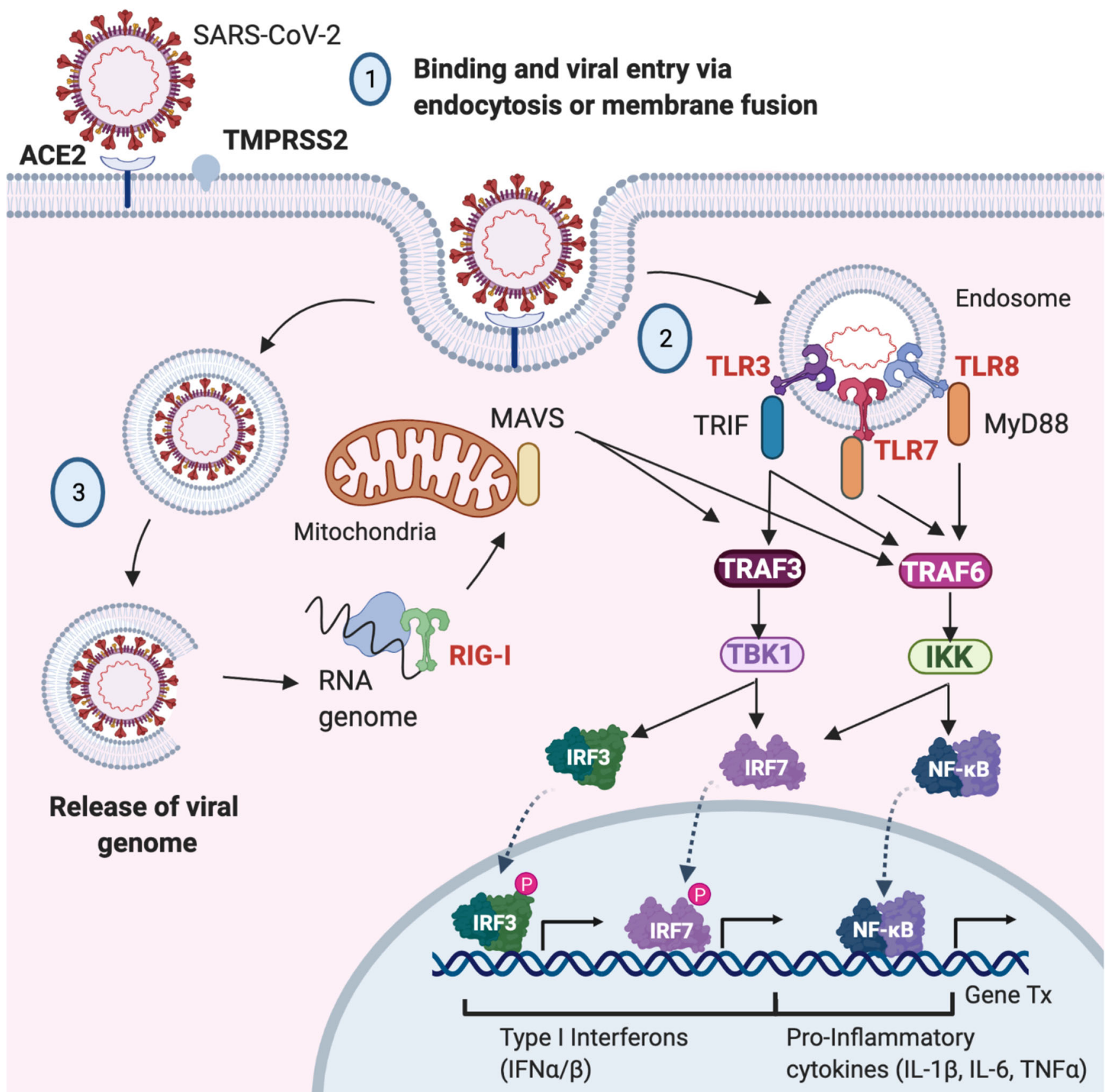

Fig. 3 Innate Immune Activation in SARS-CoV-2 Infection. The SARS$\mathrm{CoV}-2$ virus gains entry to cells by (1) binding to angiotensin converting enzyme 2 (ACE2) receptors on the cell surface through endocytosis or membrane fusion. (2) After entry the (+ sense) viral genome is released into the cytoplasm, (3) ribosomes (periwinkle) bind to viral genome to translate viral message into viral polymerase protein (light blue) and (4) RNA replication to produce (- sense) RNA genomes. A hyper inflammatory response can occur in SARS-CoV-2 infection and the presence of viral genomic and subgenomic RNA species activate cytosolic sensors such as toll-like receptor 3, 7 and 8 (TLR3/TLR7/ TKR8). The activation of TRAF-6 leads to nuclear localization of NF$\mathrm{kB}$, IRF5 and IRF7 to induce expression of interferon stimulated genes that encode antiviral proteins and pro-inflammatory cytokines. Activation of TRAF-3 causes IRF3 to assume nuclear localization to stimulate production of Type I/II interferons. Produced using BioRender.com and adapted from a template provided by Natalya Odoardi Clinical Research Coordinator, Children's Hospital of London have been implicated and described in combination with SARS-CoV-2 infection. Acute cardiac injury is associated with troponin elevation above the 99th percentile which indicates cardiac strain and injury [3]. (See Fig. 2). In a recent study, it was shown that acute heart failure patients who had acquired COVID-19 had nearly twice the risk of dying [35]. 


\section{Arrhythmias}

The development of new-onset and or progressive arrhythmia may be the first clinical sign of cardiac involvement in COVID-19 patients. A study from Wuhan examining a cohort of 137 COVID-19 patients reported that heart palpitations were among the presenting symptoms [36]. Additional reports showed increased incidence of arrhythmias in patients admitted to the ICU [23], and patients with elevated Tn exhibited higher incidence of malignant arrhythmias compared to patients with normal Tn levels [11]. Arrhythmias are a relatively common occurrence in COVID-19 patients; however, the pathophysiology behind their development is not well defined but may include direct cardiomyocyte injury, edema from pericardial infection, re-entrant arrhythmias from fibrotic scars, pro-inflammatory cytokines, and ischemia [37]. Arrhythmias that are more common in severe COVID-19 cases arise as a nonspecific clinical presentation of COVID19. Therefore, these arrhythmias are likely linked to "systemic illness rather than direct infection" [38].

\section{Myocarditis}

Myocarditis is an inflammatory disease of the myocardium that is characterized by inflammatory infiltrates and injury to heart tissue without ischemic insult [39]. In a study by Puntmann and associates., myocarditis was found to be one of the most common cardiac presentations in COVID-19 patients, as evidenced by magnetic resonance imaging (MRI) analysis [40]. This inflammation acquired during infection places additional stress on the heart even after resolution of infection [37]. Numerous studies have been directed toward illuminating the mechanisms of cardiac inflammation in COVID-19 patients and defining the extent that cardiac injury is due to direct infection by virus versus indirect inflammatory effects.

Infection of heart tissue by SARS-CoV-2 may cause nonischemic myocardial injury including acute and fulminant myocarditis and stress-induced cardiomyopathy. Cardiomyopathy may develop in COVID-19 patients that present with only mild or no respiratory symptoms. A case study of a 35-year-old male patient, with a sole cardiovascular risk factor - being overweight - presented with chest pain and fatigue. Clinical evaluation showed ECG changes but no fever or respiratory symptoms, while cardiac MRI indicated signs of acute myocarditis. The patient's test COVID-19 test was positive, and serological tests were confirmed negative for other viruses [41]. Supporting evidence for a connection between acute myocarditis and SARS-CoV-2 infection has been reported in a 53-year-old Italian woman who developed heart failure symptoms after COVID-19 diagnosis and had no previously documented heart complications [42]. In a study by Lindner and colleagues, tissues were obtained from patient autopsies to establish the presence of SARS-CoV-2 in myocardial tissue. Not only was SARS-CoV-2 present in the hearts of 24 of 39 patients, 16 of these patients had higher viral loads and also increased expression of pro-inflammatory genes when assayed with a cytokine response panel. This study documented viral presence in the myocardium [43]. Distinctions between acute and fulminant myocarditis caused by SARS-CoV-2 infection are highlighted in Fig. 4.

A case study in Brazil was the first to document SARS$\mathrm{CoV}-2$ viral particles in the heart of a child with multisystem inflammatory syndrome related to COVID-19 (MIS-C). The 11-year-old girl developed heart failure one day after admission to the hospital for MIS-C. Although she presented with systemic inflammation and multiorgan failure, heart failure was ruled the main determinant of her fatal outcome. SARS$\mathrm{CoV}-2$ viral particles were identified by electron microscopy in different heart cell lineages including cardiomyocytes, mesenchymal cells, endothelial cells, and immune cells suggesting that the myocarditis was likely due to virus infection of the heart [44]. These studies prove the difficulty in assessing whether cardiac injury is triggered directly by immune reaction to SARS-CoV-2 infection of the myocardium or due to severe systemic immune responses that can also damage cardiac tissue.

\section{Acute coronary syndrome}

Inflammation and stress can cause coronary plaque rupture or supply-demand mismatch leading to acute events such as myocardial ischemia/infarction or unstable angina [28]. These plaques must bear remarkably increased mechanical stress at particular regions, and weakening of the extracellular matrix at these sites may lead to fibrous cap rupture [45]. For example, activated macrophages secrete collagenases that degrade collagen, a major constituent of the fibrous cap on atherosclerotic plaques, which can cause plaque rupture [3]. Activated macrophages are also known to secrete tissue factor, a potent pro-coagulant that triggers thrombus formation when the plaque ruptures [3]. Addition of SARS-CoV-2 infection to acute coronary syndrome has been linked to deleterious outcomes in patients.

\section{Myocardial Infarction}

Ischemic myocardial injury can arise during severe viral infections that increase risk of plaque rupture and formation of thrombus and result in MI with ST-elevation and non-ST-elevation. ST-elevation myocardial infarction (STEMI) is caused by blockage of one of the major arteries, e.g., coronary artery of the heart and is a very serious condition. While nonST segment elevation myocardial infarction (NSTEMI) is caused by partial or temporary coronary artery blockage [46]. The type of infarct is not specific to SARS-CoV-2 infection and previously reported in hospitalized SARS [47] and 
Fig. 4 Myocarditis due to SARSCoV-2 Infection. Myocarditis can manifest in two distinct presentations and cause acute or fulminant infection of heart tissue which can lead to future longterm complications

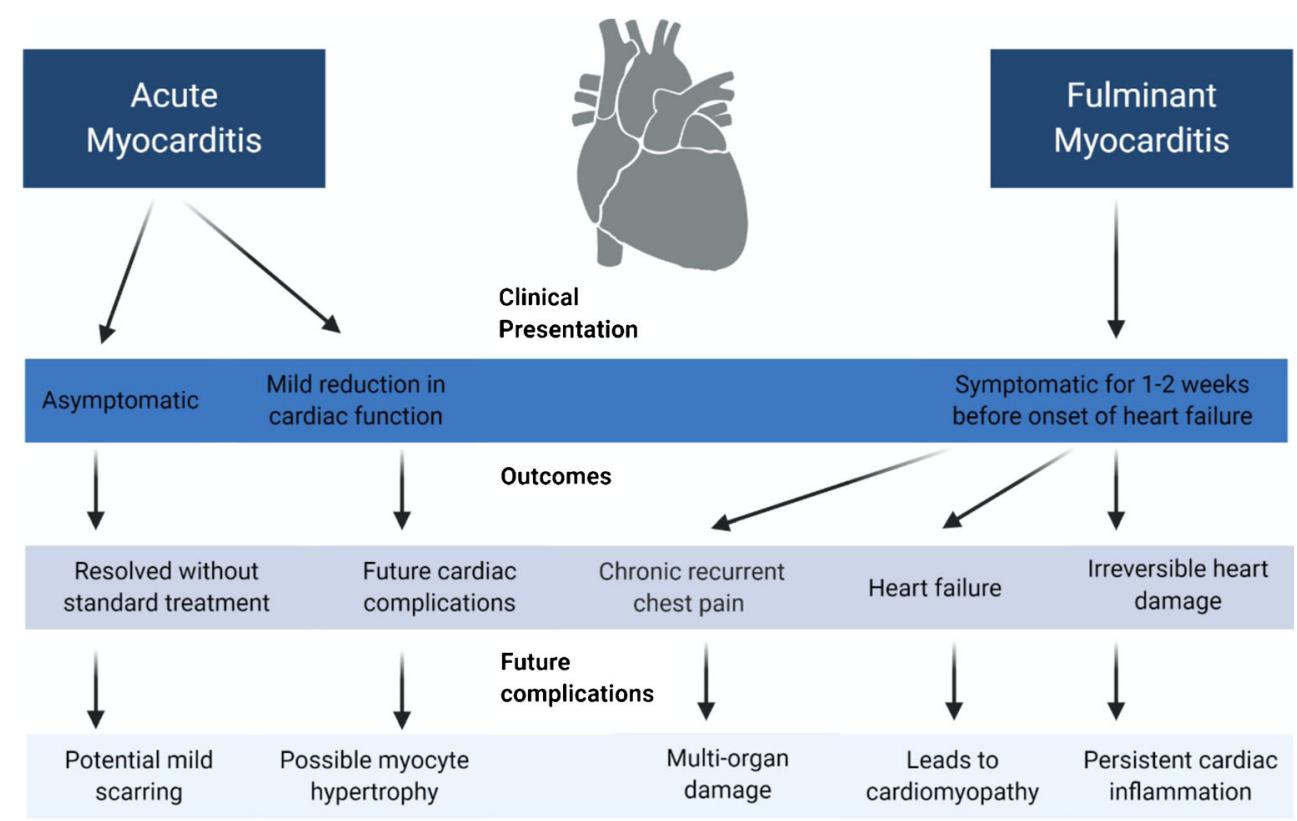

influenza patients [48]. Type I MI is myocardial injury caused by acute coronary atherothrombotic injury that occurs with either erosion or plaque rupture and frequently associated with thrombosis. The link between type I MI in COVID-19 patients has not yet been confirmed; however, additional findings may establish this connection. The risk of decreased oxygen delivery to the myocardium and vasoconstriction is inherent in SARS-CoV-2 infection and may lead to mismatch in supply and demand especially in patients with coronary heart disease. Diagnosis of MI in COVID-19 patients will have to be confirmed by clinical signals and testing and may represent a serious complication of SARS-CoV-2 infection.

Additional postmortem data or biopsies need to be collected from COVID-19 patients to ascertain whether cardiac injury is a direct result of myocardial infection. One study examining a 50year-old male in China who died of cardiac arrest revealed a lack of substantial myocardial damage. In fact, the patient had only a few sites of interstitial mononuclear inflammatory infiltrates in his hear [49]. Another study by Sala and colleagues provided more direct evidence of myocardial inflammation in a COVID19 patient seen as T-lymphocytic inflammatory infiltrates, a substantial amount of interstitial edema with limited focal necrosis, but no detectable SARS-CoV-2 genome [50]. While limited data have thwarted attempts to establish the frequency of SARS-CoV2 infection of the myocardium, it is important to further uncover sources of myocardial injury in COVID-19 patients as it contributes to substantially worsened clinical course and increased mortality.

\section{Thrombosis and Coagulation Issues}

Coagulation abnormalities are a common manifestation in severe coronavirus disease with patients also developing thromboembolisms related to coagulopathy [45]. The development of thromboembolisms may further decrease pulmonary oxygen exchange, a prevalent issue in COVID-19 patients [45]. High D-dimer levels have been detected in several patients indicating a risk for clotting abnormalities that is correlated with a six-fold increase in the requirement for mechanical ventilation [45]. In addition, elevations in D-dimer may involve modest reductions in platelet counts and increased prothrombin time and, therefore, coagulation issues. In early studies from China, elevated D-dimer levels were observed at a higher prevalence in patients with severe illness [51] and in a large percentage of patients who died [52].

A pathogenic hallmark of disseminated intravascular coagulation is dysregulated thrombin generation that is caused by heightened expression of pro-inflammatory cytokines such as IL-6 that induce expression of tissue factor on mononuclear cells that subsequently activates coagulation. While IL-6 and other pro-inflammatory cytokines are elevated in COVID-19, there is no definitive proof of thrombin generation in these patients [3]. Some patients, however, had an elevation in fibrinogen and factor VIII; therefore, they were susceptible to forming a hypercoagulable state [53]. Overall, it can be concluded that a substantial number of COVID-19 patients have coagulation abnormalities that may be below criteria established by the International Society of Thrombosis and Haemostasis. This may in fact contribute to a wide array of cardiovascular presentations.

\section{Long-Term Ramifications of SARS-CoV-2 Infection on the Heart}

Myocarditis, for example, is characterized by inflammation and myocardial injury without an ischemic cause. This 
inflammation acquired during infection adds stress to the heart even after resolution of the infection [37] and may contribute to "long-hauler syndrome" [54]. COVID-19 has also been found to "mimic an ST-elevation MI without the presence of an occluded artery." With the unknowns and unpredictability of SARS-CoV2 , limited evidence is available concerning the cardiac long-term ramifications. Studies prior to the pandemic have linked pneumonia with an increased risk of cardiovascular disease for at least ten years after recovery due to resulting lung damage [55]. Given the minimal information, previously found associations between clinical presentations in COVID-19 patients can serve as a guide for predicting future impacts. A recent article published in the NEJM discusses the onset of diabetes in patients with COVID19. This poses a serious long-term ramification as not only is the quality of life altered, but diabetes patients are also at a higher risk of serious cardiac issues from COVID-19 [56].

Researchers from Germany have been able to provide some insight into long-term impacts through a study they conducted which showed that $78 \%$ of COVID-19 patients had cardiac abnormalities evident in MRI scans two months after recovery [35]. Another study found more than the majority of recovered COVID-19 patients had ongoing myocardial inflammation [57]. A separate study found that approximately " $80 \%$ of patients with severe COVID-19 have cardiac involvement and nearly $25 \%$ have evidence of ongoing myocardial inflammation three months after diagnosis" [40]. In a published preprint, a team of researchers assessing the impact of COVID-19 on the heart used immunofluorescence to detect structural changes in cultured cardiomyocytes incubated with SARS-CoV-2 virus and found significant alterations in sarcomere structure and signs of DNA damage [58]. This finding is even more alarming given the report of heart damage in $20 \%$ of individuals with mild or no COVID19 symptoms [40]. The potential long-term impact of infection is already widespread as cases of cardiac inflammation are being reported in college athletes with minimal or no symptoms [59]. Furthermore, the true number of COVID-19 cases will likely remain unknown throughout the world. Therefore, undiagnosed or mild cases of SARS-COV-2 infection may be to blame for cardiac symptoms or injury in previously healthy individuals.

Although there have been reports of long-term cardiac consequences from SARS-CoV-2 infection, an optimistic point is that many cases of mild heart inflammation show no symptoms and can improve without treatment. While this may be the case, structured follow-up care for patients who have recovered from a severe case of COVID-19 is essential to avoid missing critical warning signs of cardiovascular disease.

\section{Treatments Strategies to Mitigate Cardiac Injury in COVID-19 Patients}

Given the alarming long-term ramifications, the Director of the National Institute of Allergies and Infectious Disease, Dr.
Anthony Fauci, expressed in a JAMA viewpoint article the vitality of therapeutics. Monitoring the clinical course of cardiac symptoms in patients who recovered from COVID-19 is critical for treating long-term ramifications of the virus [60]. Vaccines were fast tracked for FDA approval with mRNA vaccines leading the way, the Pfizer-BioNTech and Moderna vaccines are now being administered throughout the world. These vaccines meet the desired efficacy of above $80 \%$ [61]. After vaccination of a sufficient percentage of the population, rates of new infections should begin to decline. However, treatment options must also be available in the unfortunate case that the vaccine effective rates are lower than expected.

As of November 17, 2020, the FDA Coronavirus Treatment Acceleration Program has reported that the most common type of COVID-19 treatments being studied are immunomodulators. The diversity of treatments being investigated include but are not limited to gene therapies, antivirals, and neutralizing antibodies. Excessive noneffective host immune reponses to SARS-CoV-2 can be eliminated utilizing monoclonal antibodies to neutralize key immune effectors [62]. Antivirals such as remdesivir and therapies such as reconvalescent serum are not amenable to oral delivery, which reduces their suitability for controlling virus transmission [63]. On the horizon is an effective oral ribonucleoside analogue inhibitor used to inhibit influenza virus, MK-4428/ EIDD-2801, that has been found in a ferret model to reduce SARS-CoV-2 infection and block transmission of the virus [64]. Therefore, oral MK-4428/EIDD-2801 may provide an effective antiviral measure capable of countering transmission through the community [65]. Current therapies that were used consistently for cardiac issues prior to the pandemic have failed to prevent or reverse a deleterious cycle which diminishes cardiac function. A treatment that can accelerate healing or replace the damaged myocardium may be the only strategy to help patients avoid future heart failure and acute MI after COVID-19 infection. Stem cell-based therapies have taken a different approach than most therapeutics undergoing tests. Instead of targeting structural and functional adverse cardiac remodeling, these therapies regulate immune cells to promote healing of myocardial damage [66].

Anti-inflammatories such glucocorticoids may modulate inflammation-mediated inury in the lungs in COVID-19 patients. Inflammatory-based organ injury is prevalent in patients with severe COVID-19, and a subgroup of patients also exhibit marked elevations in inflammatory markers. Therapeutic strategies have been explored to counter inflammatory organ injury that manifests in viral pneumonia; however, the value of glucocorticoid treatments has remained unclear $[67,68]$. In the open-label Randomized Evaluation of COVID-19 Therapy (RECOVERY) trial, dexamethasone has been shown to reduce 28-day mortality in hospitalized COVID-19 patients receiving invasive mechanical ventilation or oxygen [69]. 
Early administration of anticoagulation therapies may be an answer to reducing cardiovascular morbidity and mortality [70]. However, a French study revealed that heparin could potentially worsen cardiac conditions for COVID-19 patients with severe disease presentation. The blood thinner heparin may increase the risk of heparin-induced thrombocytopenia (HIT). When discussing HIT in COVID-19 patients, it is important to note that it is not yet known whether this reaction is directly due to heparin administration or the virus [71]. Based on currently available evidence, COVID-19-assisted coagulopathy appears to be more "thrombotic rather than haemorrhagic" [72]. An important point of study for therapeutics is determining whether COVID-19 patients who had taken anticoagulants prior to infection have a decreased risk of mortality. Early detection and prevention of micro clotting present in COVID-19 patients could have a valuable role in therapy [73]. Autopsies conducted from deceased COVID-19 patients showed clotting in both large and small vessels [74]. Individuals have differing numbers of ACE2 receptors, which could affect how COVID-19 impacts blood clotting in response to infection. Statins are also being studied for their potential to reduce cardiovascular complications and to determine if additionally, they possess any antiviral activity. Evidence suggests that therapies lowering cholesterol such as statins could reduce viral infectivity [75].

Another type of therapeutic binds to ACE2 receptors are referred to as ACE2 soluble recombinants. ACE inhibitors have also shown to be successful in improving survival rates [76]. Multiple therapeutic strategies are being explored including targeting Notch signaling. Notch signaling is a crucial regulator of cardiovascular function and has a role in mediating viral infections [77]. Ultimately, repurposing drugs using a network medicine strategy could promote major advancements in therapeutics [78].

\section{Conclusions}

The understanding of cardiac involvement in COVID-19 patients is growing in regards to early and later stages of the illness. Reports of cardiac inflammation and indeed infection of heart cells by SARS-Co-V2 will need to be followed up by longitudinal studies and reporting of hard outcome data. The definition of myocarditis is also a source of discussion and has been diagnosed based upon myocardial biopsies, which are only performed in patients with considerable impairment of heart structure. Criteria regarding biopsies have also been shifting to now include additional immunohistological parameters [79]. We need to consider the results from current studies with that the understanding the long-term impact of COVID19 on the heart will only be revealed over time. During acute COVID-19 illness, cardiovascular damage and heart failure are more common in individuals with preexisting cardiovascular conditions that may result from hypoxia, overexertion in febrile state, and tachycardia. Whereas in later stages, COVID-related cardiac concerns can involve inflammatory myocardial involvement, heart palpitations, and persistent fatigue. Interpretation of myocardial biopsies should take these factors into consideration [80].

When assessing cardiac biomarkers on myocardial injury, it is crucial to recognize that these biomarkers are impacted by factors such as hypoxia and infection. As a result, evaluating cardiac events based on biomarkers alone is not suitable. Given the confounding variables posing troubles in evaluating data, researchers have found utilizing data from large-scale genetic association studies can help investigate associations between cardiac outcomes and SARS-CoV-2 infection. With this technique, cardiometabolic traits can be assessed to pinpoint factors leading to an increased risk of severe adverse effects from COVID-19. Overall, we have learned a great deal over the last year concerning the novel coronavirus of 2019, and we now have a much deeper appreciation of the highly variable severity of the disease and its diverse presentation. With mass scale vaccinations becoming a reality, focus on follow-up studies of individuals with history of COVID-19 infection remains crucial as well as the development of new antiviral therapies that can help further mitigate the severity of the disease.

Acknowledgements A special thank you to the American Heart Association (AHA Grant 16SDG29120002) and the FSU Office of Research Development - Collaborative Collision: Covid-19 Pandemic \#045724 for their support during this project.

\section{References}

Papers of particular interest, published recently, have been highlighted as:

- Of importance

-• Of major importance

1. Chan JF, et al. A familial cluster of pneumonia associated with the 2019 novel coronavirus indicating person-to-person transmission: a study of a family cluster. Lancet. 2020;395(10223):514-23.

2. Zhou P, Yang XL, Wang XG, Hu B, Zhang L, Zhang W, et al. A pneumonia outbreak associated with a new coronavirus of probable bat origin. Nature. 2020;579(7798):270-3.

3. Nishiga M, Wang DW, Han Y, Lewis DB, Wu JC. COVID-19 and cardiovascular disease: from basic mechanisms to clinical perspectives. Nat Rev Cardiol. 2020;17(9):543-58.

4. Zhu N, Zhang D, Wang W, Li X, Yang B, Song J, et al. A novel coronavirus from patients with pneumonia in China, 2019. N Engl J Med. 2020;382(8):727-33.

5. Bavishi C, Maddox TM, Messerli FH. Coronavirus disease 2019 (COVID-19) infection and renin angiotensin system blockers. JAMA Cardiol. 2020;5(7):745-7. 
6. Islam MR, Hoque MN, Rahman MS, Alam ASMRU, Akther M, Puspo JA, et al. Genome-wide analysis of SARS-CoV-2 virus strains circulating worldwide implicates heterogeneity. Sci Rep. 2020;10(1):14004.

7. Guzik TJ, Mohiddin SA, Dimarco A, Patel V, Savvatis K, MarelliBerg FM, et al. COVID-19 and the cardiovascular system: implications for risk assessment, diagnosis, and treatment options. Cardiovasc Res. 2020;116(10):1666-87.

8. Oudit GY, Kassiri Z, Jiang C, Liu PP, Poutanen SM, Penninger JM, et al. SARS-coronavirus modulation of myocardial ACE2 expression and inflammation in patients with SARS. Eur J Clin Investig. 2009;39(7):618-25.

9. Huertas A, et al. Endothelial cell dysfunction: a major player in SARS-CoV-2 infection (COVID-19)? Eur Respir J. 2020;56(1).

10. Prasitlumkum N, et al. Incidence of myocardial injury in COVID19-infected patients: a systematic review and meta-analysis. Diseases. 2020;8(4).

11. Guo T, Fan Y, Chen M, Wu X, Zhang L, He T, et al. Cardiovascular implications of fatal outcomes of patients with coronavirus disease 2019 (COVID-19). JAMA Cardiol. 2020;5(7):811-8.

12. Hirano T, Murakami M. COVID-19: A new virus, but a familiar receptor and cytokine release syndrome. Immunity. 2020;52(5): 731-3.

13. Shang J, Wan Y, Luo C, Ye G, Geng Q, Auerbach A, et al. Cell entry mechanisms of SARS-CoV-2. Proc Natl Acad Sci U S A. 2020;117(21):11727-34.

14. Zou X, Chen K, Zou J, Han P, Hao J, Han Z. Single-cell RNA-seq data analysis on the receptor ACE2 expression reveals the potential risk of different human organs vulnerable to 2019-nCoV infection. Front Med. 2020;14(2):185-92.

15. Pietsch $\mathrm{H}$, et al. Proof of SARS-CoV-2 genomes in endomyocardial biopsy with latency after acute infection. Int J Infect Dis. 2020;102: 70-2.

16. Tavazzi G, Pellegrini C, Maurelli M, Belliato M, Sciutti F, Bottazzi A, et al. Myocardial localization of coronavirus in COVID-19 cardiogenic shock. Eur J Heart Fail. 2020;22(5):911-5.

17. Sharma A, Garcia G Jr, Wang Y, Plummer JT, Morizono K, Arumugaswami V, et al. Human iPSC-derived cardiomyocytes are susceptible to SARS-CoV-2 infection. Cell Rep Med. 2020;1(4):100052.

18. Mehta P, McAuley D, Brown M, Sanchez E, Tattersall RS, Manson JJ, et al. COVID-19: consider cytokine storm syndromes and immunosuppression. Lancet. 2020;395(10229):1033-4.

19. Tisoncik JR, Korth MJ, Simmons CP, Farrar J, Martin TR, Katze MG. Into the eye of the cytokine storm. Microbiol Mol Biol Rev. 2012;76(1):16-32.

20. Mahallawi WH, Khabour OF, Zhang Q, Makhdoum HM, Suliman BA. MERS-CoV infection in humans is associated with a proinflammatory Th1 and Th17 cytokine profile. Cytokine. 2018;104:8-13.

21. Huang C, Wang Y, Li X, Ren L, Zhao J, Hu Y, et al. Clinical features of patients infected with 2019 novel coronavirus in Wuhan, China. Lancet. 2020;395(10223):497-506.

22. Yang X, Yu Y, Xu J, Shu H, Xia J', Liu H, et al. Clinical course and outcomes of critically ill patients with SARS-CoV-2 pneumonia in Wuhan, China: a single-centered, retrospective, observational study. Lancet Respir Med. 2020;8(5):475-81.

23. Wang D, Hu B, Hu C, Zhu F, Liu X, Zhang J, et al. Clinical characteristics of 138 hospitalized patients with 2019 novel coronavirus-infected pneumonia in Wuhan, China. JAMA. 2020;323(11):1061-9.

24. Riphagen S, Gomez X, Gonzalez-Martinez C, Wilkinson N, Theocharis P. Hyperinflammatory shock in children during COVID-19 pandemic. Lancet. 2020;395(10237):1607-8.
25. Kang Y, Chen T, Mui D, Ferrari V, Jagasia D, Scherrer-Crosbie M, et al. Cardiovascular manifestations and treatment considerations in COVID-19. Heart. 2020;106(15):1132-41.

26. Bonow RO, Fonarow GC, O'Gara PT, Yancy CW. Association of coronavirus disease 2019 (COVID-19) with myocardial injury and mortality. JAMA Cardiol. 2020;5(7):751-3.

27. Zheng YY, Ma YT, Zhang JY, Xie X. COVID-19 and the cardiovascular system. Nat Rev Cardiol. 2020;17(5):259-60.

28. Bavishi C, Bonow RO, Trivedi V, Abbott JD, Messerli FH, Bhatt DL. Special article - acute myocardial injury in patients hospitalized with COVID-19 infection: a review. Prog Cardiovasc Dis. 2020;63(5):682-9.

29. Ssentongo P, Ssentongo AE, Heilbrunn ES, Ba DM, Chinchilli VM. Association of cardiovascular disease and 10 other preexisting comorbidities with COVID-19 mortality: a systematic review and meta-analysis. PLoS One. 2020;15(8):e0238215.

30. Clerkin KJ, Fried JA, Raikhelkar J, Sayer G, Griffin JM, Masoumi A, et al. COVID-19 and cardiovascular disease. Circulation. 2020;141(20):1648-55.

31. Ceriello A, et al. Issues for the management of people with diabetes and COVID-19 in ICU. Cardiovasc Diabetol. 2020;19(1):114.

32. Rebello CJ, Kirwan JP, Greenway FL. Obesity, the most common comorbidity in SARS-CoV-2: is leptin the link? Int J Obes. 2020;44(9):1810-7.

33. Unudurthi SD, Luthra P, Bose RJC, McCarthy JR, Kontaridis MI. Cardiac inflammation in COVID-19: Lessons from heart failure. Life Sci. 2020;260:118482.

34. Lala A, Johnson KW, Januzzi JL, Russak AJ, Paranjpe I, Richter F, et al. Prevalence and impact of myocardial injury in patients hospitalized with COVID-19 infection. J Am Coll Cardiol. 2020;76(5): $533-46$

35. Doolub G, et al. Impact of COVID-19 on inpatient referral of acute heart failure: a single-centre experience from the south-west of the UK. ESC Heart Fail. 2021.

36. Liu K, Fang YY, Deng Y, Liu W, Wang MF, Ma JP, et al. Clinical characteristics of novel coronavirus cases in tertiary hospitals in Hubei Province. Chin Med J. 2020;133(9):1025-31.

37. Siripanthong B, Nazarian S, Muser D, Deo R, Santangeli P, Khanji MY, et al. Recognizing COVID-19-related myocarditis: the possible pathophysiology and proposed guideline for diagnosis and management. Heart Rhythm. 2020;17(9):1463-71.

38. Bhatla A, Mayer MM, Adusumalli S, Hyman MC, Oh E, Tierney A, et al. COVID-19 and cardiac arrhythmias. Heart Rhythm. 2020;17(9):1439-44.

39. Esfandiarei M, McManus BM. Molecular biology and pathogenesis of viral myocarditis. Annu Rev Pathol. 2008;3:127-55.

40. Puntmann VO, Carerj ML, Wieters I, Fahim M, Arendt C, Hoffmann J, et al. Outcomes of cardiovascular magnetic resonance imaging in patients recently recovered from coronavirus disease 2019 (COVID-19). JAMA Cardiol. 2020;5(11):1265-73.

41. Paul JF, Charles P, Richaud C, Caussin C, Diakov C. Myocarditis revealing COVID-19 infection in a young patient. Eur Heart J Cardiovasc Imaging. 2020;21(7):776.

42. Inciardi RM, Lupi L, Zaccone G, Italia L, Raffo M, Tomasoni D, et al. Cardiac involvement in a patient with coronavirus disease 2019 (COVID-19). JAMA Cardiol. 2020;5(7):819-24.

43. Lindner D, Fitzek A, Bräuninger H, Aleshcheva G, Edler C, Meissner K, et al. Association of cardiac infection with SARSCoV-2 in confirmed COVID-19 autopsy cases. JAMA Cardiol. 2020;5(11):1281-5.

44. Dolhnikoff M, Ferreira Ferranti J, de Almeida Monteiro RA, Duarte-Neto AN, Soares Gomes-Gouvêa M, Viu Degaspare N, et al. SARS-CoV-2 in cardiac tissue of a child with COVID-19related multisystem inflammatory syndrome. Lancet Child Adolesc Health. 2020;4(10):790-4. 
45. Levi M, Thachil J, Iba T, Levy JH. Coagulation abnormalities and thrombosis in patients with COVID-19. Lancet Haematol. 2020;7(6): $438-40$.

46. Warren-Gash C, Hayward AC, Hemingway H, Denaxas S, Thomas $\mathrm{SL}$, Timmis AD, et al. Influenza infection and risk of acute myocardial infarction in England and Wales: a CALIBER selfcontrolled case series study. J Infect Dis. 2012;206(11):1652-9.

47. Peiris JS, et al. Clinical progression and viral load in a community outbreak of coronavirus-associated SARS pneumonia: a prospective study. Lancet. 2003;361(9371):1767-72.

48. Kwong JC, Schwartz KL, Campitelli MA. Acute myocardial infarction after laboratory-confirmed influenza infection. N Engl J Med. 2018;378(26):2540-1.

49. Xu Z, Shi L, Wang Y, Zhang J, Huang L, Zhang C, et al. Pathological findings of COVID-19 associated with acute respiratory distress syndrome. Lancet Respir Med. 2020;8(4):420-2.

50. Sala S, Peretto G, Gramegna M, Palmisano A, Villatore A, Vignale $\mathrm{D}$, et al. Acute myocarditis presenting as a reverse Tako-Tsubo syndrome in a patient with SARS-CoV-2 respiratory infection. Eur Heart J. 2020;41(19):1861-2.

51. Guan WJ, Ni ZY, Hu Y, Liang WH, Ou CQ, He JX, et al. Clinical characteristics of coronavirus disease 2019 in China. N Engl J Med. 2020;382(18):1708-20.

52. Zhou F, Yu T, du R, Fan G, Liu Y, Liu Z, et al. Clinical course and risk factors for mortality of adult inpatients with COVID-19 in Wuhan, China: a retrospective cohort study. Lancet. 2020;395(10229):1054-62.

53. Panigada M, Bottino N, Tagliabue P, Grasselli G, Novembrino C, Chantarangkul V, et al. Hypercoagulability of COVID-19 patients in intensive care unit: a report of thromboelastography findings and other parameters of hemostasis. J Thromb Haemost. 2020;18(7): 1738-42.

54. Rubin R. As their numbers grow, COVID-19 "Long Haulers" Stump Experts. JAMA. 2020.

55. Corrales-Medina VF, Alvarez KN, Weissfeld LA, Angus DC, Chirinos JA, Chang CCH, et al. Association between hospitalization for pneumonia and subsequent risk of cardiovascular disease. JAMA. 2015;313(3):264-74.

56. Rubino F, Amiel SA, Zimmet P, Alberti G, Bornstein S, Eckel RH, et al. New-onset diabetes in COVID-19. N Engl J Med. 2020;383(8):789-90.

57. Huang L, et al. Cardiac involvement in patients recovered from COVID-2019 identified using magnetic resonance imaging. JACC Cardiovasc Imaging. 2020;13(11):2330-9.

58. Perez-Bermejo JA et al. SARS-CoV-2 infection of human iPSCderived cardiac cells predicts novel cytopathic features in hearts of COVID-19 patients. bioRxiv, 2020.

59. Rajpal S, et al. Cardiovascular magnetic resonance findings in competitive athletes recovering from COVID-19 infection. JAMA Cardiol. 2020.

60. Kim PS, Read SW, Fauci AS. Therapy for early COVID-19: a critical need. JAMA. 2020;324(21):2149-50.

61. Anderson RM, Vegvari C, Truscott J, Collyer BS. Challenges in creating herd immunity to SARS-CoV-2 infection by mass vaccination. Lancet. 2020;396(10263):1614-6.

62. Shah VK, Firmal P, Alam A, Ganguly D, Chattopadhyay S. Overview of immune response during SARS-CoV-2 infection: lessons from the past. Front Immunol. 2020;11:1949.
63. Humeniuk R, Mathias A, Cao H, Osinusi A, Shen G, Chng E, et al. Safety, tolerability, and pharmacokinetics of remdesivir, an antiviral for treatment of COVID-19, in healthy subjects. Clin Transl Sci. 2020;13(5):896-906.

64. Toots $\mathrm{M}$, et al. Characterization of orally efficacious influenza drug with high resistance barrier in ferrets and human airway epithelia. Sci Transl Med. 2019;11(515).

65. Cox RM, Wolf JD, Plemper RK. Therapeutically administered ribonucleoside analogue MK-4482/EIDD-2801 blocks SARS-CoV2 transmission in ferrets. Nat Microbiol. 2020.

66. Wagner MJ, Khan M, Mohsin S. Healing the broken heart; the immunomodulatory effects of stem cell therapy. Front Immunol. 2020;11:639.

67. Russell CD, Millar JE, Baillie JK. Clinical evidence does not support corticosteroid treatment for 2019-nCoV lung injury. Lancet. 2020;395(10223):473-5.

68. Shang L, Zhao J, Hu Y, du R, Cao B. On the use of corticosteroids for 2019-nCoV pneumonia. Lancet. 2020;395(10225):683-4.

69. Group RC, et al. Dexamethasone in hospitalized patients with COVID-19 - preliminary report. N Engl J Med. 2020.

70. Tremblay D, van Gerwen M, Alsen M, Thibaud S, Kessler A, Venugopal S, et al. Impact of anticoagulation prior to COVID-19 infection: a propensity score-matched cohort study. Blood. 2020;136(1):144-7.

71. Daviet F, Guervilly C, Baldesi O, Bernard-Guervilly F, Pilarczyk E, Genin A, et al. Heparin-induced thrombocytopenia in severe COVID-19. Circulation. 2020;142(19):1875-7.

72. Waghmare A, Abidi MZ, Boeckh M, Chemaly RF, Dadwal S, el Boghdadly Z, et al. Guidelines for COVID-19 management in hematopoietic cell transplantation and cellular therapy recipients. Biol Blood Marrow Transplant. 2020;26(11):1983-94.

73. Connors JM, Levy JH. COVID-19 and its implications for thrombosis and anticoagulation. Blood. 2020;135(23):2033-40.

74. Rapkiewicz AV, Mai X, Carsons SE, Pittaluga S, Kleiner DE, Berger JS, et al. Megakaryocytes and platelet-fibrin thrombi characterize multi-organ thrombosis at autopsy in COVID-19: a case series. EClinicalMedicine. 2020;24:100434.

75. Radenkovic D, et al. Cholesterol in relation to COVID-19: should we care about it? J Clin Med. 2020:9(6).

76. Batlle D, Wysocki J, Satchell K. Soluble angiotensin-converting enzyme 2: a potential approach for coronavirus infection therapy? Clin Sci (Lond). 2020;134(5):543-5.

77. Rizzo P, Vieceli Dalla Sega F, Fortini F, Marracino L, Rapezzi C, Ferrari R. COVID-19 in the heart and the lungs: could we "Notch" the inflammatory storm? Basic Res Cardiol. 2020;115(3):31.

78. Sehirli AO, Sayiner S, Serakinci N. Role of melatonin in the treatment of COVID-19; as an adjuvant through cluster differentiation 147 (CD147). Mol Biol Rep. 2020;47(10):8229-33.

79. Caforio AL, et al. Current state of knowledge on aetiology, diagnosis, management, and therapy of myocarditis: a position statement of the European Society of Cardiology Working Group on Myocardial and Pericardial Diseases. Eur Heart J. 2013;34(33): 2636-48, 2648a-2648d.

80. Puntmann V, Nagel E. Cardiac involvement after recovering from COVID-19-reply. JAMA Cardiol. 2020.

Publisher's Note Springer Nature remains neutral with regard to jurisdictional claims in published maps and institutional affiliations. 